\title{
Nutritional Impact of Canola Meal on Performance, Blood Constituents and Immune Response of Broilers
}

\author{
A. A. Ghazalah ${ }^{1}$, A. M. El-Kaiaty ${ }^{1}$, Hady F. A. Motawe ${ }^{2}$ \& A. S. Radwan ${ }^{2}$ \\ ${ }^{1}$ Animal Production Department, Faculty of Agriculture, Cairo University, Giza, Egypt \\ ${ }^{2}$ Regional Centre for Food and Feed, Agricultural Research Centre, Giza, Egypt \\ Correspondence: A. S. Radwan, Regional Centre for Food and Feed, Agricultural Research Centre, Giza, Egypt. \\ E-mail: mrahmed_2010sad@yahoo.com
}

Received: August 20, 2020

Accepted: November 25, 2020

Online Published: December 15, 2020

doi:10.5539/jas.v13n1p135

URL: https://doi.org/10.5539/jas.v13n1p135

\begin{abstract}
This study was conducted to evaluate the effects of replacing soybean meal (SBM) protein with canola meal (CM) protein on productive performance, nutrient digestibility, immune response, lymphoid organs, blood parameters, carcass fatty acids and cecum microbiota of broilers. A total of 160 one d-old Arbor Acres broiler chicks were randomly allocated to 4 dietary treatments of 5 replicates, where, CM protein replaced SBM protein at $0,30,60$, and $90 \%$ for a 39 days feeding trial. The results showed no significant differences in productive performance parameters among control, $30 \%$ and $60 \%$ treatment groups, while, at $90 \%$ replacement level, all values decreased $(\mathrm{P}<0.0001)$ all over the experimental period. The $90 \%$ replacement group showed depression of crude protein $(\mathrm{P}<0.001)$ and crude fiber $(\mathrm{P}<0.001)$ digestibility and spleen relative weight $(\mathrm{P}=0.0386)$ with increase of thymus $(\mathrm{P}=0.0555)$, bursa $(\mathrm{P}=0.0334)$ and thyroid relative weight $(\mathrm{P}=0.0276)$ as well as thyroid hormones $(\mathrm{P}=0.0034,0339)$ for $\mathrm{T}_{3}, \mathrm{~T}_{4}$, respectively, while, there were no significant differences among control, $30 \%$ and $60 \%$ treatment groups for those criteria. However, CM levels had no effect on serum haemagglutination inhibition (HI) titer against Newcastle disease. CM significantly decreased serum cholesterol content $(\mathrm{P}=0.0002)$ while increased HDL $(\mathrm{P}=0.0532)$, compared to the control. $\mathrm{CM}$ levels showed an increase in carcass meat content of unsaturated fatty acids content $(\mathrm{P}<0.0001)$ as the replacement level gradually increased. Erucic acid did not detected in carcass. All CM levels decreased cecum content of E. coli $(\mathrm{P}=0.0051)$ while increased that of Lactobacillus $(\mathrm{P}=4094)$. Conclusively, $\mathrm{CM}$ can be used safely in broiler diet to replace up to $60 \%$ of SBM protein without negative effects on growth and immune response of broilers.
\end{abstract}

Keywords: broilers, canola meal, digestibility, fatty acids, growth, immunity, microbiota

\section{Introduction}

The poultry feeding costs represent approximately $70 \%$ of the overall costs of poultry production. Most countries of the world consider soybean meal (SBM) is the main source of protein in poultry diets (Husak et al., 2008) as in Egypt. Lack of availability of the local soybean and SBM with their highly prices, beside to increasing of globally prices of SBM led to encourage the producers of poultry feeds to use alternatives like cottonseed meal (Aftab, 2009; Yuan et al., 2014), sunflower meal (Aftab, 2009; Moghaddam et al., 2012), cassava leaf meal (Iheukwumere et al., 2008), moringa leaf meal (Gadzirayi et al., 2012; Abbas, 2013) and flaxseed meal (Tarek et al., 2015) otherwise, their combinations. At the same time, the declining profit of poultry companies in light of highly prices of the main raw feedstuffs forced them to search for cheaper alternative protein. If CM used as an alternative protein for SBM in broiler diets may be able to provide liberation to the broiler and soybean industry. According to the USDA (2014), the rapeseed production including canola varieties ranks second among oilseed crops worldwide. On the other hand, Canola Council of Canada (2015) reported that, CM has a widely utilizing as an alternative protein source for soybean meal (SBM) in poultry diets where it has a good balance of essential amino acids. According to Newkirk (2009), the CM contains approximately $40 \%$ crude protein, has a good-balanced amino acid profile and high levels of sulfur-containing amino acids compared with SBM.

Although CM glucosinolates have antibacterial, antifungal properties, and cancer-chemoprevention activity, their anti-nutritional effects have limited the use of meals from rapeseed for human food and animal feed (Szydlowska-Czerniak et al., 2011). Besides, canola oil is rich in unsaturated fatty acids like omega 3 fatty acids. 
The fatty acid composition of poultry meat is an important quality parameter especially with respect to potentially affecting human health via poultry meat consumption (Mantzioris et al., 2000; Rahimi et al., 2011).

The nutritive value of CM is inferior compared to SBM in chicken diets due to its content of some anti-nutritive factors, like non-starch polysaccharides (NSP) that account for 18-20\% and high fiber content which represents $11-12 \%$ (Khajali \& Slominski, 2012) that led to low energy availability content compared to SBM for poultry. Accordingly, CM could not be used as $100 \%$ alternative for SBM in poultry diets (Khajali \& Slominski, 2012). Therefore, the objective of the present study was to evaluate the effects of replacing SBM protein with CM protein at $0,30,60$ and $90 \%$ on performance, nutrients digestibility, immune response, lymphoid organs, blood parameters, carcass fatty acids and cecum microbiota of broilers.

\section{Materials and Methods}

The Institutional Animal Care and Use Committee of Cairo University approved the experimental protocol used in this study, with approval number CU-II-F-22-20 on May 2020.

\subsection{Experimental Chicks, Design and Management}

This experiment was carried out at Regional Centre for Food and Feed, Agricultural Research Centre, Ministry of Agriculture, Giza, Egypt. A total of one hundred and sixty day-old-chicks male Arbor Acres broiler chicks were randomly distributed into four treatment groups of five replicates (battery cages) each of 8 chicks using a completely randomized design. Treatment groups were fed diets contain canola meal (CM) at 0, 30, 60 and 90\% of SBM protein (Table 1).

Diets were formulated in the Regional Center for Food and Feed to be isonitrogenous, isocaloric and mycotoxins-free, as levels of aflatoxins and other mycotoxins were below the detection limit (detection limit 1 ppb) (Ross et al., 1997) as well as free from any medication or antibiotics. The diets were formulated to meet the nutrient requirements of broiler chicks during starting, growing and finishing periods according to Arbor Acres plus Broiler Nutrition Specifications (2014).

All chicks were fed a starter diet from day one to 14 days of age. From day 15 to 28 days of age, the birds were switched to a grower diet, and from day 29 to 39 days of age, chicks were fed a finisher diet. The composition and determind chemical analyses of the used diets are stated in Table 1. Feed and water were available ad-libitum during the experimental period (39 days). Water was provided by drip nipples. At the first week, Temperature was adjusted at $30 \pm 0.5^{\circ} \mathrm{C}$ then lowered $2{ }^{\circ} \mathrm{C}$ each successive week, and then maintained at $24 \pm 0.5$ ${ }^{\circ} \mathrm{C}$. Relative humidity was about $60 \%$ to $70 \%$ at the first week of age then, lowered to $50-60 \%$ from the $2^{\text {nd }}$ week of age until the end of experiment. The broiler chicks were exposed to $23 \mathrm{hrs} \mathrm{light} \mathrm{and} 1$ hour dark during the day at the first week. From the second week up to the end of the experiment, the light was $20 \mathrm{hrs}$ and $4 \mathrm{hrs}$ dark during the day.

The chicks had an average initial body weight of $46.00 \pm 1.00 \mathrm{~g}$. Chicks were vaccinated against IB at $6^{\text {th }}$ day of age, $\mathrm{H}_{5} \mathrm{~N}_{1}$ at $9^{\text {th }}$ day, Gumboro D78 at $13^{\text {th }}$ and $24^{\text {th }}$ days of age. Weekly, body weight (BW) and feed intake (FI) were recorded for each replicate during all periods of growth. The feed conversion ratio (FCR) was calculated by dividing feed intake by body weight gain.

\subsection{Digestibility Trial}

At 39 day of age, a digestibility trial was conducted for 3 days collection period using 5 birds within each treatment. Birds were individually housed in metabolic cages and the total collection method cited by Abou-Raya and Galal (1971) was executed to determine the apparent digestibility (AD) of nutrients. Nitrogen $(\mathrm{N})$, ether extract (EE), crude fiber (CF), and ash content of dried excreta as well as those of feed were determined according to AOAC (2016) using the N. 928.08, 2003.06, 2011.25 and 920.153 methods, respectively. The total protein content was calculated using Kjeldahl nitrogen and a conversion factor of 6.25. The following equation indicates how to determine $\% \mathrm{AD}$ :

$$
\% \mathrm{AD}=[(\mathrm{f}-\mathrm{e}) / \mathrm{f}] \times 100
$$

Where, $f$ and $e$ are the intake and excreted nutrient in grams, respectively.

\subsection{Immune Response and Lymphoid Organs}

To determine the immune response of chicks, whole sheep blood collected in heparinized tube was washed three times in phosphate buffered saline (PBS, $\mathrm{pH} 7.4)$ and diluted in PBS to 25 per cent $\left(\mathrm{v} \mathrm{v}^{-1}\right)$. Ten chicks from each treatment group (2 from each replicate) were immunized with $1 \mathrm{~mL}$ of 25 per cent sheep red blood cells (SRBCs) (Kundu et al., 1999) in thigh muscles at day 28. Booster dose of SRBCs antigen was given at day 35. Blood samples were collected at day 35 of age for assessing hemagglutination (HA) titer (Abdel-Ati et al., 1984) 
against SRBCs by using freshly prepared one per cent SRBCs. At 39 days of age, another blood samples were collected randomly from wing vein of 5 birds, from each treatment group (one bird/replicate). Serum samples were subjected to haemagglutination inhibition (HI) test for determining antibody titers against Newcastle disease (ND) vaccine as described by Swayne et al. (1998). In addition, the differences in relative weight of lymphoid organs including spleen, bursa and thymus, other than the relaive weight of thyroid gland were detected.

Table 1. Composition and calculated analysis of the experimental diets

\begin{tabular}{|c|c|c|c|c|c|c|c|c|c|c|c|c|}
\hline \multirow{3}{*}{ Ingredients $(\%)$} & \multicolumn{4}{|c|}{ Starter (1-14 days) } & \multicolumn{4}{|c|}{ Grower (15-28 days) } & \multicolumn{4}{|c|}{ Finisher (29-39 days) } \\
\hline & \multicolumn{12}{|c|}{ Replacement levels (\%) } \\
\hline & 0 & 30 & 60 & 90 & 0 & 30 & 60 & 90 & 0 & 30 & 60 & 90 \\
\hline Yellow corn $(7.35 \%)$ & 59.00 & 54.71 & 50.41 & 46.05 & 61.21 & 57.90 & 54.01 & 50.01 & 61.48 & 57.48 & 53.13 & 48.73 \\
\hline Soybean meal $(45.32 \%)$ & 27.29 & 19.10 & 10.91 & 2.73 & 23.14 & 16.20 & 9.25 & 2.32 & 25.00 & 17.50 & 10.00 & 2.50 \\
\hline Canola meal (34.11\%) & 0 & 10.88 & 21.75 & 32.63 & 0 & 9.22 & 18.45 & 27.67 & 0 & 9.97 & 19.93 & 29.89 \\
\hline Corn gluten $(60.8 \%)$ & 8.23 & 8.91 & 9.30 & 9.30 & 9.69 & 10.00 & 10.50 & 11.00 & 6.00 & 6.50 & 7.10 & 7.70 \\
\hline Vegetable oil & 0.97 & 2.13 & 3.50 & 5.00 & 1.70 & 2.64 & 3.86 & 5.18 & 3.73 & 5.00 & 6.41 & 7.85 \\
\hline Di calcium phosphate & 2.01 & 1.97 & 1.94 & 1.89 & 1.81 & 1.76 & 1.73 & 1.69 & 1.63 & 1.59 & 1.54 & 1.50 \\
\hline Lime stone & 0.89 & 0.85 & 0.79 & 0.74 & 0.81 & 0.77 & 0.72 & 0.68 & 0.66 & 0.61 & 0.56 & 0.52 \\
\hline DL-methionine (98\%) & 0.31 & 0.15 & 0.09 & 0.05 & 0.23 & 0.09 & 0.05 & 0.02 & 0.25 & 0.10 & 0.05 & 0.02 \\
\hline L-lysine-HCL (98\%) & 0.60 & 0.61 & 0.62 & 0.91 & 0.52 & 0.53 & 0.54 & 0.55 & 0.38 & 0.39 & 0.40 & 0.41 \\
\hline Common salt & 0.30 & 0.30 & 0.30 & 0.30 & 0.32 & 0.32 & 0.32 & 0.32 & 0.32 & 0.32 & 0.32 & 0.32 \\
\hline Vit./Min. Premix* & 0.30 & 0.30 & 0.30 & 0.30 & 0.30 & 0.30 & 0.30 & 0.30 & 0.30 & 0.30 & 0.30 & 0.30 \\
\hline Choline Chloride & 0.10 & 0.10 & 0.10 & 0.10 & 0.27 & 0.27 & 0.27 & 0.27 & 0.26 & 0.26 & 0.26 & 0.26 \\
\hline \multirow[t]{2}{*}{ Total } & 100 & 100 & 100 & 100 & 100 & 100 & 160 & 100 & 100 & 100 & 100 & 100 \\
\hline & \multicolumn{12}{|c|}{ Calculated analyses } \\
\hline ME $\left(\mathrm{Kcal} \mathrm{kg}^{-1}\right)$ & 2988 & 2982 & 3004 & 3006 & 3121 & 3102 & 3100 & 3104 & 3208 & 3202 & 3205 & 3210 \\
\hline Methionine \% & 0.70 & 0.58 & 0.56 & 0.56 & 0.62 & 0.52 & 0.51 & 0.51 & 0.60 & 0.48 & 0.48 & 0.48 \\
\hline Lysine \% & 1.44 & 1.44 & 1.44 & 1.65 & 1.29 & 1.29 & 1.29 & 1.29 & 1.19 & 1.19 & 1.19 & 1.19 \\
\hline \multirow[t]{2}{*}{ Met + Cys \% } & 1.08 & 1.08 & 1.18 & 1.29 & 0.99 & 0.99 & 1.08 & 1.18 & 0.94 & 0.94 & 1.04 & 1.15 \\
\hline & \multicolumn{12}{|c|}{ \% Determined chemical analyses } \\
\hline $\mathrm{CP}$ & 22.33 & 22.33 & 22.24 & 22.25 & 21.38 & 21.25 & 21.26 & 21.26 & 19.85 & 19.79 & 19.82 & 19.85 \\
\hline $\mathrm{CF}$ & 3.11 & 3.73 & 4.33 & 4.91 & 2.90 & 3.39 & 4.00 & 4.50 & 2.88 & 3.50 & 4.10 & 4.65 \\
\hline $\mathrm{EE}$ & 3.30 & 5.25 & 7.50 & 9.70 & 4.00 & 5.70 & 7.60 & 9.70 & 5.85 & 8.00 & 10.15 & 12.40 \\
\hline $\mathrm{Ca}$ & 0.85 & 0.86 & 0.85 & 0.85 & 0.76 & 0.76 & 0.76 & 0.77 & 0.67 & 0.67 & 0.67 & 0.67 \\
\hline Avail. P. & 0.48 & 0.46 & 0.45 & 0.43 & 0.44 & 0.42 & 0.41 & 0.39 & 0.41 & 0.39 & 0.37 & 0.36 \\
\hline
\end{tabular}

Note. * Vitamins-minerals mixture supplied per kg of diet: vit. (A), 12000 I.U., vit. ( $\mathrm{D}_{3}$ ), 2000 I.U; vit. (E), $10 \mathrm{mg}$; vit. $\left(\mathrm{K}_{3}\right), 2 \mathrm{mg}$; vit. $\left(\mathrm{B}_{1}\right), 1 \mathrm{mg}$; vit. $\left(\mathrm{B}_{2}\right), 5 \mathrm{mg}$; vit. $\left(\mathrm{B}_{6}\right), 1.5 \mathrm{mg}$; vit. $\left(\mathrm{B}_{12}\right), 10 \mu \mathrm{g}$; Biotin, $50 \mu \mathrm{g}$; Pantothenic acid, 10mg; Niacin, $30 \mathrm{mg}$; Folic acid, $1 \mathrm{mg}$; Manganese, $60 \mathrm{mg}$; Zinc, $50 \mathrm{mg}$; Iron, $30 \mathrm{mg}$; Copper, $10 \mathrm{mg}$; Iodine, $1 \mathrm{mg}$; Selenium, $0.1 \mathrm{mg}$ and Cobalt, $0.1 \mathrm{mg}$.

\subsection{Blood Parameters}

At the end of experiment, blood samples were taken from wing vein of 5 chickens in each group and directly aliquoted into 2-mL sterile vials and allowed to clot for $4 \mathrm{~h}$. After centrifugation (10 min, $2000 \mathrm{rpm})$, the serum was collected and stored at $-20{ }^{\circ} \mathrm{C}$ until the time of analysis. The serum samples were assigned for determination of total protein (TP), liver enzymes including aspartate aminotransferase (AST), alanine aminotransferase (ALT), as well as kidney function indices including urea and creatinine and cholesterol using commercially available kits (Biosystem S.A., Costa Brava, 30, Barcelona, Spain) according to manufacturer's instructions. Serum thyroid hormones concentration were determined by 125 I labelled RIA kits for $\mathrm{T}_{3}$ (IM1699, Immunotech, Czech Republic) and $\mathrm{T}_{4}$ (IM1447 Immunotech, Czech Republic) (Okuliarova et al., 2011).

\subsection{Cecal Contents and Fatty Acids Profile of Carcass Meat}

Five birds from each treatment group were slaughtered at $39 \mathrm{~d}$ of age. Then, cecal contents were taken for bacterial counting (Collin et al., 1995). Fatty acids profile of overall carcass meat was determined by extracting total lipids from about 5 grams of collected samples in a homogenizer with $20 \mathrm{ml}$ of 2:1 chloroform-methanol and then filtered through Whatman No. 1 filter paper (Folch et al., 1957). The fatty acids were determined as methyl esters using Gas Liquid chromatography technique according to AOAC (2016). Saturated and unsaturated fatty acids of meat were expressed as percentage of total fatty acids. 


\subsection{Statistical Analyses}

A polynomial regression analysis was used to predict the effect of the inclusion of various levels of canola meal in the diet on the parameters of growth performance, nutrient digestibility, immune response, lymphoid organs, biochemical parameters, fatty acids profile of carcass meat and cecum microbial content. The polynomial regression models were selected based on the significance of the regression coefficients $(P<0.05)$ and on the value of the coefficient of determination. Data obtained in this study were analyzed by one-way analysis of variance using the SAS software general linear model (SAS, 2004). The main factor was canola meal replacement level. Mean values were compared using the Duncan's New Multiple Range test (Duncan, 1955) when significant differences existed. The fixed effects model used in the analysis was as follow: $Y_{i j}=\mu+T_{i}+e_{i j}$, Where, $Y_{\mathrm{ij}}$ : the observation of the $\mathrm{J}_{\text {th }}$ chick in the $\mathrm{i}_{\mathrm{th}}$ treatment; $\mu$ : the overall mean; $\mathrm{T}_{\mathrm{i}}$ : effect of the $\mathrm{i}_{\text {th }}$ treatment $(i=1,2,3,4)$ and $e_{i j}$ : the random error effect. The significance level was set at $(\alpha=0.05)$.

\section{Results}

\subsection{Productive Performance}

Results presented in Table 2, indicated presence of differences in body weight and body weight gain/period among experimental groups. Body weight and body weight gain of the group that fed $90 \% \mathrm{CM}$ diet were significantly lower at all ages compared to the other experimental groups and the control. However, both $30 \%$ and $60 \% \mathrm{CM}$ diets groups are nearly similar to the control group without significant difference.

Feed intake of the groups fed 30 and $60 \%$ CM substituted from SBM protein were not significantly differ, however, at $90 \%$ replacement level, FCR was significantly inferior, compared to the control.

Table 2. Effect of replacing Soybean meal protein by canola meal protein on productive performance

\begin{tabular}{|c|c|c|c|c|c|c|}
\hline Items & Control & $\mathrm{CM}(30 \%)$ & $\mathrm{CM}(60 \%)$ & CM $(90 \%)$ & SEM & P-value \\
\hline \multicolumn{7}{|l|}{$0-14 d$} \\
\hline BW, g & $408.86^{\mathrm{a}}$ & $400.66^{\mathrm{a}}$ & $393.00^{\mathrm{a}}$ & $322.80^{\mathrm{b}}$ & 6.31 & $<0.0001$ \\
\hline BWG, g & $363.46^{\mathrm{a}}$ & $355.46^{\mathrm{a}}$ & $346.26^{\mathrm{a}}$ & $276.73^{b}$ & 6.20 & $<0.0001$ \\
\hline FI, g & $430.86^{\mathrm{a}}$ & $417.33^{\mathrm{ab}}$ & $410.00^{\mathrm{b}}$ & $346.66^{c}$ & 5.24 & $<0.0001$ \\
\hline FCR & $1.18^{\mathrm{a}}$ & $1.18^{\mathrm{a}}$ & $1.18^{\mathrm{a}}$ & $1.25^{b}$ & 0.02 & 0.0454 \\
\hline \multicolumn{7}{|l|}{$15-28 d$} \\
\hline BW, g & $1471.13^{\mathrm{a}}$ & $1470.33^{\mathrm{a}}$ & $1456.27^{\mathrm{a}}$ & $1047.73^{b}$ & 12.21 & $<0.0001$ \\
\hline BWG, g & $1062.27^{\mathrm{a}}$ & $1069.67^{\mathrm{a}}$ & $1063.27^{\mathrm{a}}$ & $724.93^{\mathrm{b}}$ & 11.82 & $<0.0001$ \\
\hline FI, g & $1390.00^{\mathrm{a}}$ & $1386.67^{\mathrm{ab}}$ & $1366.87^{b}$ & $1229.33^{c}$ & 7.66 & $<0.0001$ \\
\hline FCR & $1.31^{\mathrm{a}}$ & $1.29^{\mathrm{a}}$ & $1.28^{\mathrm{a}}$ & $1.69^{\mathrm{b}}$ & 0.02 & $<0.0001$ \\
\hline \multicolumn{7}{|l|}{$29-39 d$} \\
\hline $\mathrm{BW}, \mathrm{g}$ & $2498.67^{\mathrm{a}}$ & $2490.27^{\mathrm{a}}$ & $2470.00^{\mathrm{a}}$ & $1979.40^{\mathrm{b}}$ & 18.30 & $<0.0001$ \\
\hline BWG, g & $1027.53^{\mathrm{a}}$ & $1019.93^{\mathrm{a}}$ & $1013.73^{\mathrm{a}}$ & $931.66^{\mathrm{b}}$ & 18.09 & 0.0011 \\
\hline FI, g & 1770.00 & 1792.33 & 1795.33 & 1822.33 & 31.75 & 0.7141 \\
\hline FCR & $1.72^{\mathrm{a}}$ & $1.75^{\mathrm{ab}}$ & $1.77^{\mathrm{b}}$ & $1.95^{\mathrm{c}}$ & 0.01 & $<0.0001$ \\
\hline \multicolumn{7}{|l|}{$0-39 d$} \\
\hline BWG, g & $2453.27^{\mathrm{a}}$ & $2445.07^{\mathrm{a}}$ & $2423.27^{\mathrm{a}}$ & $1933.33^{b}$ & 18.19 & $<0.0001$ \\
\hline FI, g & $3590.87^{\mathrm{a}}$ & $3596.33^{a}$ & $3572.20^{\mathrm{a}}$ & $3398.33^{b}$ & 33.98 & $<0.0001$ \\
\hline FCR & $1.46^{\mathrm{a}}$ & $1.47^{\mathrm{a}}$ & $1.47^{\mathrm{a}}$ & $1.75^{\mathrm{b}}$ & 0.01 & $<0.0001$ \\
\hline
\end{tabular}

Note. $\mathrm{CM}=$ Canola meal, $\mathrm{BW}=$ body weight, $\mathrm{BWG}=$ body weight gain, $\mathrm{FI}=$ feed intake, $\mathrm{FCR}=$ feed conversion ratio; ${ }^{\mathrm{a}-\mathrm{b}}$ Means with different superscripts within each row are significantly different, SEM: standard error of the mean, $\mathrm{P}$-value $=$ significance level at $\alpha=0.05$. Regression equations set for $(0-14 \mathrm{~d}): \mathrm{BW}=421.21-0.89 \mathrm{x}, \mathrm{R}^{2}=$ $0.50, \mathrm{P}$-value $<0.0001, \mathrm{SE}=0.11 ; \mathrm{BWG}=375.89-0.90 \mathrm{x}, \mathrm{R}^{2}=0.53, \mathrm{P}$-value $<0.0001, \mathrm{SE}=0.11 ; \mathrm{FI}=440.21-$ $0.87 \mathrm{x}, \mathrm{R}^{2}=0.59, \mathrm{P}$-value $<0.0001, \mathrm{SE}=0.95 ; \mathrm{FCR}=1.14+0.001 \mathrm{x}, \mathrm{R}^{2}=0.56, \mathrm{P}$-value $<0.0001, \mathrm{SE}=0.0001$. For $(15-28 \mathrm{~d}): \mathrm{BW}=1554.007-4.281 \mathrm{x}, \mathrm{R}^{2}=0.58, \mathrm{P}$-value $<0.0001, \mathrm{SE}=0.468 ; \mathrm{BWG}=1132.79-3.39 \mathrm{x}, \mathrm{R}^{2}=$ $0.55, \mathrm{P}$-value $<0.0001, \mathrm{SE}=0.40 ; \mathrm{FI}=1428.99-1.82 \mathrm{x}, \mathrm{R}^{2}=0.67, \mathrm{P}$-value $<0.0001, \mathrm{SE}=0.17 ; \mathrm{FCR}=1.235+$ $0.004 \mathrm{x}, \mathrm{R}^{2}=0.46, \mathrm{P}$-value $<0.0001, \mathrm{SE}=0.0005$. For $(29-39 \mathrm{~d})$ : $\mathrm{BW}=2586.69-5.33 \mathrm{x}, \mathrm{R}^{2}=0.64$, $\mathrm{P}$-value $<$ $0.0001, \mathrm{SE}=0.53 ; \mathrm{BWG}=1032.69-1.05 \mathrm{x}, \mathrm{R}^{2}=0.70, \mathrm{P}$-value $<0.0001, \mathrm{SE}=0.09 ; \mathrm{FCR}=1.749+0.002 \mathrm{x}, \mathrm{R}^{2}=$ $0.59, \mathrm{P}$-value $<0.0001, \mathrm{SE}=0.0002$. For $(0-39 \mathrm{~d}): \mathrm{BWG}=2541.37-5.34 \mathrm{x}, \mathrm{R}^{2}=0.64, \mathrm{P}$-value $<0.0001, \mathrm{SE}=$ $0.53 ; \mathrm{FI}=3675.89-2.67 \mathrm{x}, \mathrm{R}^{2}=0.31, \mathrm{P}$-value $<0.0001, \mathrm{SE}=0.52 ; \mathrm{FCR}=1.434+0.003 \mathrm{x}, \mathrm{R}^{2}=0.56, \mathrm{P}$-value $<$ $0.0001, \mathrm{SE}=0.0003$. 


\subsection{Nutrient Digestibility}

No significant differences were observed among CM levels for the average values of organic matter (OM), EE and nitrogen free extract (NFE) digestibility (Table 3). Data showed that the highest CM inclusion level had the lowest crude protein $(\mathrm{CP})$ and $\mathrm{CF}$ digestibility, being 82.7 and $22.6 \%$, respectively. The corresponding values of the control were 84.9 and $24.9 \%$, respectively.

Table 3. Effect of replacing of Soybean meal protein by canola meal protein on nutrients digestibility of experimental finisher diets

\begin{tabular}{|c|c|c|c|c|c|}
\hline \multirow{2}{*}{ Canola meal level } & \multicolumn{5}{|c|}{ Nutrients apparent digestibility (\%) } \\
\hline & $\mathrm{OM}$ & $\mathrm{CP}$ & $\mathrm{EE}$ & $\mathrm{CF}$ & NFE \\
\hline 0 & 86.4 & $84.9^{\mathrm{a}}$ & 73.3 & $24.9^{\mathrm{a}}$ & 85.9 \\
\hline 30 & 86.1 & $85.1^{\mathrm{a}}$ & 73.3 & $24.7^{\mathrm{a}}$ & 86.0 \\
\hline 60 & 86.2 & $84.7^{\mathrm{a}}$ & 73.6 & $23.8^{\mathrm{ab}}$ & 85.0 \\
\hline 90 & 86.1 & $82.7^{\mathrm{b}}$ & 72.5 & $22.6^{\mathrm{b}}$ & 84.8 \\
\hline SEM & 0.39 & 0.39 & 0.79 & 0.42 & 0.23 \\
\hline P-value & 0.535 & $<0.001$ & 0.166 & $<0.001$ & 0.285 \\
\hline
\end{tabular}

Note. ${ }^{\mathrm{a}-\mathrm{b}}$ Means with different superscripts within each column are significantly different, SEM: standard error of the mean, $\mathrm{P}$-value $=$ significance level at $\alpha=0.05 ; \mathrm{OM}=$ organic matter, $\mathrm{CP}=$ crude protein, $\mathrm{EE}=$ ether extract, $\mathrm{CF}$ $=$ crude fiber, $\mathrm{NFE}=$ nitrogen free extract. Regression equations set for: $\mathrm{CP}=85.40-0.02 \mathrm{x}, \mathrm{R}^{2}=0.59, \mathrm{P}$-value $=$ $0.0033, \mathrm{SE}=0.006 ; \mathrm{CF}=25.17-0.03 \mathrm{x}, \mathrm{R}^{2}=0.82, \mathrm{P}$-value $<0.0001, \mathrm{SE}=0.004$.

\subsection{Immune Response and Lymphoid Organs}

The Haemagglutination Inhibition (HI) titer against ND virus did not differ $(\mathrm{P}>0.05)$ at 39 days of age due to the replacement of soybean meal by $\mathrm{CM}$ in the broilers diets (Table 4). Also, there were no significant differences $(\mathrm{P}>0.05)$ among all groups in response of SRBCs injection.

Increasing level of CM up to $90 \%$ of SBM protein significantly lowered percentage of spleen, while, increased those of thymus, bursa and thyroid gland, compared to the control. While, no significant differences were found between groups fed either 30 or $60 \% \mathrm{CM}$ and the control group fed diet based only on SBM.

Table 4. Effect of replacing of Soybean meal protein by canola meal protein on immune response and lymphoid organs

\begin{tabular}{lllllll}
\hline Items & Control & CM $(30 \%)$ & CM (60\%) & CM (90\%) & SEM & P-value \\
\hline ND titer & 6.66 & 7.66 & 8.66 & 8.33 & 0.66 & 0.2341 \\
SRBCs titer & 6.44 & 6.44 & 5.66 & 5.66 & 0.42 & 0.3605 \\
Thyroid (\%) & $0.08^{\mathrm{b}}$ & $0.09^{\mathrm{b}}$ & $0.08^{\mathrm{b}}$ & $0.16^{\mathrm{a}}$ & 0.013 & 0.0276 \\
Spleen (\%) & $0.09^{\mathrm{a}}$ & $0.10^{\mathrm{a}}$ & $0.09^{\mathrm{a}}$ & $0.07^{\mathrm{b}}$ & 0.014 & 0.0386 \\
Bursa (\%) & $0.12^{\mathrm{b}}$ & $0.13^{\mathrm{b}}$ & $0.13^{\mathrm{b}}$ & $0.15^{\mathrm{a}}$ & 0.018 & 0.0334 \\
Thymus (\%) & $0.31^{\mathrm{b}}$ & $0.26^{\mathrm{b}}$ & $0.45^{\mathrm{ab}}$ & $0.61^{\mathrm{a}}$ & 0.093 & 0.0555 \\
\hline
\end{tabular}

Note. $\mathrm{CM}=$ Canola meal, $\mathrm{ND}=$ Newcastle disease, $\mathrm{SRBC}$ 's $=$ sheep red blood cells; ${ }^{\mathrm{a}-\mathrm{b}}$ Means with different superscripts within each row are significantly different, SEM: standard error of the mean, P-value $=$ significance level at $\alpha=0.05$. Regression equations set for: Thyroid $(\%)=0.0650+0.0008 \mathrm{x}, \mathrm{R}^{2}=0.64, \mathrm{P}$-value $<0.0001, \mathrm{SE}=$ 0.0001 ; Spleen $(\%)=0.0985-0.0003 x, R^{2}=0.36, \mathrm{P}$-value $=0.0012, \mathrm{SE}=0.00007$; Bursa $(\%)=0.1112+0.0008 \mathrm{x}$, $\mathrm{R}^{2}=0.47, \mathrm{P}$-value $=0.0002, \mathrm{SE}=0.0002$; Thymus $(\%)=0.245+0.004 \mathrm{x}, \mathrm{R}^{2}=0.77, \mathrm{P}$-value $<0.0001, \mathrm{SE}=$ 0.0004 .

\subsection{Blood Biochemical Parameters}

All the levels tend to decrease serum AST $(P=0.0071)$, Creatinine $(P<0.0001)$ and urea $(P=0.0007)$ values compared to the control (Table 5). In addition, no significant differences were recorded among treatment groups in regard to the serum content of total protein, albumin and globulin values. Serum content of thyroid hormones including $\mathrm{T}_{3}(\mathrm{P}=0.0034)$ and $\mathrm{T}_{4}(\mathrm{P}=0.0339)$ were higher in chicks fed $\mathrm{CM}$ at $90 \%$ of SBM protein compared to control group fed the basal diet (Table 5). Moreover, chicks fed diets contain CM recorded lower values of 
cholesterol $(\mathrm{P}<0.05)$ and LDL cholesterol but not significantly $(\mathrm{P}>0.05)$, compared to the control group fed diet based on SBM only. As the control group recorded the lowest value of HDL cholesterol $(\mathrm{P}=0.0532)$, accordingly, the HDL/LDL ratio increased gradually $(\mathrm{P}<0.05)$ as the replacement level of $\mathrm{CM}$ increased (Table $5)$.

Table 5. Effect of replacing of Soybean meal protein by canola meal protein on biochemical parameters

\begin{tabular}{lllllll}
\hline Items & Control & CM $(30 \%)$ & CM $(60 \%)$ & CM $(90 \%)$ & \pm SEM & P-value \\
\hline Total Protein $(\mathrm{g} / \mathrm{dL})$ & 3.26 & 3.30 & 2.90 & 3.13 & 0.15 & 0.1680 \\
Albumin $(\mathrm{g} / \mathrm{dL})$ & 2.13 & 2.16 & 2.00 & 2.16 & 0.05 & 0.1131 \\
Globulin $(\mathrm{g} / \mathrm{dL})$ & 1.13 & 1.14 & 0.90 & 0.97 & 0.17 & 0.7613 \\
AST $(\mathrm{U} / \mathrm{L})$ & $119.00^{\mathrm{a}}$ & $93.33^{\mathrm{b}}$ & $90.33^{\mathrm{b}}$ & $85.00^{\mathrm{b}}$ & 5.16 & 0.0071 \\
ALT $(\mathrm{U} / \mathrm{L})$ & $46.00^{\mathrm{a}}$ & $37.00^{\mathrm{b}}$ & $33.66^{\mathrm{b}}$ & $39.66^{\mathrm{ab}}$ & 2.07 & 0.0165 \\
Creatinine (mg/dL) & $2.10^{\mathrm{a}}$ & $1.20^{\mathrm{b}}$ & $1.20^{\mathrm{b}}$ & $1.20^{\mathrm{b}}$ & 0.09 & $<0.0001$ \\
Urea (mg/dL) & $7.50^{\mathrm{a}}$ & $4.00^{\mathrm{b}}$ & $2.50^{\mathrm{b}}$ & $2.20^{\mathrm{b}}$ & 0.58 & 0.0007 \\
$\mathrm{~T}_{3}(\mathrm{ng} / \mathrm{dL})$ & $188.33^{\mathrm{c}}$ & $208.33^{\mathrm{bc}}$ & $230.00^{\mathrm{b}}$ & $262.00^{\mathrm{a}}$ & 9.55 & 0.0034 \\
$\mathrm{~T}_{4}(\mu \mathrm{dL})$ & $3.5^{\mathrm{b}}$ & $3.86^{\mathrm{ab}}$ & $4.30^{\mathrm{a}}$ & $4.46^{\mathrm{a}}$ & 0.19 & 0.0339 \\
Cholesterol (mg/dL) & $150.33^{\mathrm{a}}$ & $128.00^{\mathrm{b}}$ & $125.00^{\mathrm{b}}$ & $76.66^{\mathrm{c}}$ & 6.03 & 0.0002 \\
HDL (mg/dL) & $83.33^{\mathrm{b}}$ & $97.00^{\mathrm{a}}$ & $96.00^{\mathrm{a}}$ & $98.66^{\mathrm{a}}$ & 2.403 & 0.0532 \\
LDL (mg/dL) & 20.00 & 19.00 & 18.66 & 17.66 & 2.09 & 0.8860 \\
HDL/LDL & $4.16^{\mathrm{b}}$ & $5.10^{\mathrm{a}}$ & $5.14^{\mathrm{a}}$ & $5.59^{\mathrm{a}}$ & 0.57 & 0.0491 \\
\hline
\end{tabular}

Note. $\mathrm{CM}=$ Canola meal; ${ }^{\mathrm{a}-\mathrm{c}}$ Means with different superscripts within each row are significantly different, SEM: standard error of the mean, $\mathrm{P}$-value $=$ significance level at $\alpha=0.05$. Regression equations set for: $\mathrm{AST}=112.67-$ $0.35 \mathrm{x}, \mathrm{R}^{2}=0.65, \mathrm{P}$-value $=0.0015, \mathrm{SE}=0.08 ; \mathrm{ALT}=42.67-0.09 \mathrm{x}, \mathrm{R}^{2}=0.38, \mathrm{P}$-value $=0.0312, \mathrm{SE}=0.03$; Creatinine $=1.83-0.009 x, R^{2}=0.60, P$-value $=0.0031, \mathrm{SE}=0.002 ;$ Urea $=6.66-0.06 \mathrm{x}, \mathrm{R}^{2}=0.84, \mathrm{P}$-value $<$ $0.0001, \mathrm{SE}=0.008 ; \mathrm{T}_{3}=185.76+0.81 \mathrm{x}, \mathrm{R}^{2}=0.94, \mathrm{P}$-value $<0.0001, \mathrm{SE}=0.06 ; \mathrm{T}_{4}=3.53+0.01 \mathrm{x}, \mathrm{R}^{2}=0.75$, $\mathrm{P}$-value $=0.0003, \mathrm{SE}=0.002 ;$ Cholesterol $=153.60-0.75 \mathrm{x}, \mathrm{R}^{2}=0.77, \mathrm{P}$-value $<0.0001, \mathrm{SE}=0.12 ; \mathrm{HDL}=$ $87.00+0.15 \mathrm{x}, \mathrm{R}^{2}=0.66, \mathrm{P}$-value $=0.0013, \mathrm{SE}=0.03 ; \mathrm{HDL} / \mathrm{LDL}=4.35+0.01 \mathrm{x}, \mathrm{R}^{2}=0.86, \mathrm{P}$-value $<0.0001$, $\mathrm{SE}=0.002$.

\subsection{Fatty Acids Profile of Carcass Meat and Secum Microbial Content}

Results indicated highly significant differences $(\mathrm{P}<0.0001)$ in fatty acids contents of carcass meat among experimental groups (Table 6).

Chicks fed diets with different levels of CM showed significantly increase in carcass meat content of the unsaturated fatty acids (UFA), particularly palmitolic and oleic as mono UFA with higher values for treatments 30 and $60 \% \mathrm{CM}$ of SBM protein. Collectively, total saturated fatty acids (SFA) showed lower values by feeding $\mathrm{CM}$ at different levels, compared to the group fed basal diet.

Accordingly, the UFA/SFA ratio showed an evident increase in CM treatment groups, compared to the group fed basal diet. Data in Table 6 indicate that the linoleic (n-6) fatty acid is higher in chicks fed 60 or $90 \%$ CM instead SBM protein by about $77 \%$ over the control fed basal diet and by about $21 \%$ over those fed CM at $30 \%$ replacement level. Erucic acid did not detected in carcass meat of all treatment groups.

It is worthy to note that the lowest $(\mathrm{P}<0.05)$ Enterococcus and E. coli count had been detected for chicks having $\mathrm{CM}$ at 30\% replacement of SBM protein (Table 6). Salmonella microbes had not been detected in cecum in all experimental groups. 
Table 6. Effect of replacing of Soybean meal protein by canola meal protein on fatty acids profile of carcass meat ( $\%$ of total fatty acids) and cecum microbial content ( $\log 10 \mathrm{CFU} / \mathrm{g}$ digesta)

\begin{tabular}{lllllll}
\hline Items & Control & CM $(30 \%)$ & CM $(60 \%)$ & CM $(90 \%)$ & SEM & P-value \\
\hline Myristic & $3.82^{\mathrm{a}}$ & $0.58^{\mathrm{c}}$ & $0.60^{\mathrm{c}}$ & $0.85^{\mathrm{b}}$ & 0.02 & $<0.0001$ \\
Palmitic & $27.85^{\mathrm{a}}$ & $23.80^{\mathrm{b}}$ & $24.06^{\mathrm{b}}$ & $23.14^{\mathrm{c}}$ & 0.18 & $<0.0001$ \\
Palmitolic & $2.54^{\mathrm{b}}$ & $4.00^{\mathrm{a}}$ & $4.00^{\mathrm{a}}$ & $1.97^{\mathrm{c}}$ & 0.03 & $<0.0001$ \\
Stearic & $11.03^{\mathrm{a}}$ & $7.75^{\mathrm{c}}$ & $6.77^{\mathrm{d}}$ & $8.46^{\mathrm{b}}$ & 0.09 & $<0.0001$ \\
Oleic & $28.50^{\mathrm{d}}$ & $39.19^{\mathrm{a}}$ & $35.88^{\mathrm{b}}$ & $33.66^{\mathrm{c}}$ & 0.14 & $<0.0001$ \\
Linoleic (n-6) & $13.00^{\mathrm{c}}$ & $19.23^{\mathrm{b}}$ & $23.01^{\mathrm{a}}$ & $23.29^{\mathrm{a}}$ & 0.11 & $<0.0001$ \\
Linolenic (n-3) & $0.80^{\mathrm{c}}$ & $1.27^{\mathrm{b}}$ & $2.07^{\mathrm{a}}$ & $2.05^{\mathrm{a}}$ & 0.04 & $<0.0001$ \\
SFA & $42.70^{\mathrm{a}}$ & $32.13^{\mathrm{b}}$ & $31.43^{\mathrm{b}}$ & $32.45^{\mathrm{b}}$ & 0.29 & $<0.0001$ \\
UFA & $44.84^{\mathrm{c}}$ & $63.69^{\mathrm{a}}$ & $64.96^{\mathrm{a}}$ & $60.97^{\mathrm{b}}$ & 0.32 & $<0.0001$ \\
n-6/n-3 & $16.25^{\mathrm{a}}$ & $15.14^{\mathrm{a}}$ & $11.12^{\mathrm{b}}$ & $11.36^{\mathrm{b}}$ & 0.27 & $<0.0001$ \\
UFA/SFA & $0.99^{\mathrm{b}}$ & $1.98^{\mathrm{a}}$ & $2.07^{\mathrm{a}}$ & $1.88^{\mathrm{a}}$ & 0.22 & $<0.0001$ \\
Erucic & $\mathrm{ND}$ & $\mathrm{ND}$ & $\mathrm{ND}$ & $\mathrm{ND}^{\mathrm{a}}$ & - & - \\
Lact. Count & 7.60 & 7.77 & 7.71 & 7.86 & 0.10 & 0.4094 \\
En. Count & $6.29^{\mathrm{a}}$ & $4.94^{\mathrm{b}}$ & $6.31^{\mathrm{a}}$ & $6.43^{\mathrm{a}}$ & 0.22 & 0.0051 \\
E. coli & $5.19^{\mathrm{a}}$ & $3.26^{\mathrm{c}}$ & $4.44^{\mathrm{b}}$ & $4.80^{\mathrm{ab}}$ & 0.128 & $<0.0001$ \\
Salmonella & $\mathrm{ND}$ & $\mathrm{ND}$ & $\mathrm{ND}$ & $\mathrm{ND}$ & - & - \\
\hline Note. CM & & & & & \\
\hline
\end{tabular}

Note. $\mathrm{CM}=$ Canola meal; ${ }^{\mathrm{a}-\mathrm{d}}$ Means with different superscripts within each row are significantly different, SEM: standard error of the mean, $\mathrm{P}$-value $=$ significance level at $\alpha=0.05, \mathrm{ND}=$ not detected. Regression equations set for: Myristic $=2.47-0.02 \mathrm{x}, \mathrm{R}^{2}=0.44, \mathrm{P}$-value $=0.0261, \mathrm{SE}=0.009 ;$ Palmitic $=26.55-0.04 \mathrm{x}, \mathrm{R}^{2}=0.64$, P-value $=0.0033, \mathrm{SE}=0.01 ;$ Palmitolic $=2.98+0.01 \mathrm{x}, \mathrm{R}^{2}=0.60, \mathrm{P}$-value $=0.0032, \mathrm{SE}=0.005 ;$ Stearic $=9.81-0.03 \mathrm{x}$, $\mathrm{R}^{2}=0.38, \mathrm{P}$-value $=0.0334, \mathrm{SE}=0.012 ;$ Oleic $=31.7+0.1 \mathrm{x}, \mathrm{R}^{2}=0.60, \mathrm{P}$-value $=0.0031, \mathrm{SE}=0.03 ;$ Linoleic $=$ $14.8+0.1 \mathrm{x}, \mathrm{R}^{2}=0.85, \mathrm{P}$-value $<0.0001, \mathrm{SE}=0.02 ;$ Linolenic $=0.88+0.01 \mathrm{x}, \mathrm{R}^{2}=0.86, \mathrm{P}$-value $<0.0001, \mathrm{SE}=$ $0.002 ; \mathrm{SFA}=39.40-0.03 \mathrm{x}, \mathrm{R}^{2}=0.57, \mathrm{P}$-value $=0.0045, \mathrm{SE}=0.03 ; \mathrm{UFA}=51.18+0.17 \mathrm{x}, \mathrm{R}^{2}=0.47, \mathrm{P}$-value $=$ $0.0138, \mathrm{SE}=0.06 ; \mathrm{n}-6 / \mathrm{n}-3=16.30-0.06 \mathrm{x}, \mathrm{R}^{2}=0.76, \mathrm{P}$-value $=0.0002, \mathrm{SE}=0.01 ; \mathrm{UFA} / \mathrm{SFA}=1.360+0.009 \mathrm{x}, \mathrm{R}^{2}$ $=0.50, \mathrm{P}$-value $=0.0100, \mathrm{SE}=0.003 ; E n$. Count $=5.89-0.01 \mathrm{x}, \mathrm{R}^{2}=0.62, \mathrm{P}$-value $=0.0024, \mathrm{SE}=0.003 ; E$. coli $=$ $4.61-0.02 \mathrm{x}, \mathrm{R}^{2}=0.61, \mathrm{P}$-value $=0.0028, \mathrm{SE}=0.005$.

\section{Discussion}

\subsection{Productive Performance}

In the present study, FI of the group that fed $90 \% \mathrm{CM}$ diet was lowest compared with other experimental groups in starting, growing and allover period. This could be attributed to several reasons. The higher glucosinolates content of $90 \% \mathrm{CM}$ group compared to other experimental groups comes first (Khajali \& Slominski, 2012) which caused palatability reduction and may interfere with the function of thyroid gland causing drastic disturbance of thyroid hormones resulting in negative effects on growth performance particularly on BWG. Therefore, increasing of serum $\mathrm{T}_{4}$ and $\mathrm{T}_{3}$ concentrations in our study may also led to reduction of the broilers' BWG. Furthermore, CM at higher level caused an increase of thyroid size and its metabolic activities which led to energy and other nutrients utilization for maintenance rather than growth (Woyengo et al., 2011). The second reason is higher dietary fiber content of $90 \% \mathrm{CM}$ group compared to other experimental groups (Woyngo et al., 2011), causing low energy as well as low protein digestibility (Khajali \& Slominski, 2012; Bovera et al., 2014; Gopinger et al., 2014), that might have reduced the performance of chicks. The third reason is higher content of phenolic compounds such as condensed tannins of $90 \% \mathrm{CM}$ group compared to other experimental groups (Mansoori \& Acamovic, 2007) which can decrease the bioavailability of protein by forming complexes with protein and proteolytic enzymes in the gastrointestinal tract (Khajali \& Slominski, 2012). Finally, the higher NSP of $90 \% \mathrm{CM}$ group compared to other experimental groups accounted one of possible reasons for growth performance's impairing which represent $18-20 \%$ (Khajali \& Slominski, 2012), that caused an increase in the viscosity of digesta and reduced the digestion as well as the absorption of nitrogen and subsequently resulting in poor growth performance (Mushtaq et al., 2007); all contribute to reduced feed intake in the $90 \% \mathrm{CM}$ diet which is reflected on BWG and Final BW depression. The results are in agreement with previous studies that found an adverse effect on FI of broiler chickens fed diets contained CM (Payvastegan et al., 2013; Gopinger et al., 2014; Aljuobori et al., 2016; Zhang \& Adeola, 2017). 
In the same vein, CM contains higher phytic acid (2.9-3.2\%) than SBM which contains only 1.4\% (Payvastegan et al., 2013) that leading to non-full availability of some nutrients which mainly are amino acids, calcium and phosphorus, for example, reduction of lysine digestibility of CM compared to SBM (Mushtaq et al., 2007). According to our results, the broilers that fed $90 \% \mathrm{CM}$ as replacement of SBM protein showed an inferior FCR.

\subsection{Nutrient Digestibility}

In the present study, the digestibility of $\mathrm{CP}$ and $\mathrm{CF}$ was inversely related to the used level of $\mathrm{CM}$ in the diet. This means that both $\mathrm{CP}$ and $\mathrm{CF}$ digestion coefficient tended to decrease $(\mathrm{P}<0.05)$ as the $\mathrm{CM}$ replacement level increased more than $60 \%$ of SBM protein. Such decrease could be attributed to that CM contains some biologically active chemical compounds such as phytate, NSP and tannins which can reduce the digestibility of nutrients (Khajali \& Slominski, 2012). Dietary fiber reduces nutrient digestibility due to its physiochemical properties, leading to a more rapid rate of passage that limits the amount of time available for nutrient breakdown (Thacker \& Petri, 2011). Moreover, total dietary fiber values for canola meal are higher than those of SBM due to a much higher content of lignin with associated polyphenols (Khajali \& Slominski, 2012). As expected, an increase in the level of inclusion of $\mathrm{CM}$ results in an increase in the level of fiber in the diet. Accordingly, this increase decreases the protein digestibility. In this respect, Pustjens et al. (2013) reported that NSP are not degraded by poultry endogenous enzymes, and thus increase gut viscosity and consequently reduce nutrient utilization. Consistent with the present study, Pinheiro et al. (2008) observed significant differences in the nutrient digestibility of dry matter (DM) and CP between the diets of broilers with low CF and the diets of broilers with high CF. In addition, Landero et al. (2012); Gopinger et al. (2014); Toghyani et al. (2017) observed a decrease in digestibility of $\mathrm{CP}$ with increased canola meal inclusion in a diet for weaned piglets and broilers, respectively, and attributed this decrease to the high CF content of canola meal.

\subsection{Immune Response and Lymphoid Organs}

Feeding CM had no significant effect on HI titer against ND virus, that as the same as the results obtained by Mushtaq et al. (2007) and that obtained by An et al. (2016). These results are contrary with results obtained by Ahmad et al. (2007); who reported that HI antibody titer against ND virus was lowest in diets containing 10\% $\mathrm{CM}$, whereas, it was highest in those having $15 \% \mathrm{CM}$. In another respect, $\mathrm{CM}$ at $30 \%$ and $60 \%$ replacement level did not cause significant effect on the relative weight of lymphoid organs compared to the control which agree with results reported by Ahmed et al. (2015) when they fed broilers diets contained 5, 10 and 20\% CM. Also, the present results are compatible with the results of Ashnie et al. (2015) where, they did not found a significant differences of relative spleen weights among broilers fed diets contained $0,7.5$ and $15 \% \mathrm{CM}$ while, the broilers fed diets contained 22.5 and 30\% CM showed a significant increase of relative weights of spleen compared to the control group which was contrary with our results. In this respect, Taraz et al. (2006) reached to $38.2,28.95$ and $23.16 \% \mathrm{CM}$ as maximum levels in starter, grower and finisher diets, respectively; they reported no significant differences in relative spleen weights between all CM groups and the CM free group; that showed disagreement with the present results of broilers fed $90 \%$ CM replacement of SBM compared to control, as the starter, grower and finisher diets contained 32.63, 27.67 and 29.89\% CM, respectively. In the same vein, An et al. (2016) reported no significant differences among relative spleen weights of broilers fed wheat-SBM based diets contained $0,3,5,10$ and $15 \% \mathrm{CM}$. It is well known that spleen, bursa and thymus are considered a part of the secondary and primary lymphoid organs (H. Lillehoj \& E. Lillehoj, 2000) responsible for producing cells that protect the birds from the invaded microorganisms.

\subsection{Blood Biochemical Parameters}

Regard to thyroid hormones, the higher concentration of $\mathrm{T}_{3}$ in the $\mathrm{CM}$ diets may be attributed to effects of glucosinolate hydrolysis products on peripheral $\mathrm{T}_{4}$ monodeiodination. In line with our results, Newkirk and Classen (2002); Kermanshahi and Abbasipour (2006); Maroufyan and Kermanshahi (2006); Aljuobori et al. (2016) have stated that $\mathrm{CM}$ feeding elevated $\mathrm{T}_{3}$ level in broiler chickens. On the other hand, Woyengo et al. (2011) reported that serum $T_{4}$ was increased while serum $T_{3}$ not affected significantly by the substitution of $C M$. In this respect, Aljuobori et al. (2016) suggested that glucosinolates in CM may destroy cellular $\mathrm{T}_{3}$ receptors and thus increase the thyroid hormones level in blood. In contrary to our results, Taraz et al. (2006); Mikulski et al. (2012); Payvastegan et al. (2017) indicated that an increasing of inclusion levels of CM was followed by plasma $\mathrm{T}_{3}$ concentrations reduction. However, Taraz et al. (2006); Payvastegan et al. (2017) reported no significant differences in plasma $T_{4}$ among all experimental groups. They attributed these discrepancies to differences in glucosinolates content in CM, diet ingredients, composition of gut microorganism besides, the extent of glucosinolates degradation by heat during the oil extraction process (Woyengo et al., 2011). 
Obtained results indicated that feeding CM to broiler reduced cholesterol level in blood serum, which is a desirable outcome for consumers. In this connection, Mnisi and Mlambo (2018) with quail and El-Medany and El-Reffaei (2015) with growing rabbits attained the same result. In contrary with our results, Payvastegan et al. (2017) reported no significant differences in plasma concentrations of cholesterol and HDL cholesterol in broilers receiving diets containing different levels of CM up to 30\%. In the same line Payvastegan et al. (2013) reported no significant differences in plasma concentrations of cholesterol and HDL in broilers receiving diets containing different levels of solvent extracted $\mathrm{CM}$ up to $20 \%$. The higher HDL fraction $(\mathrm{P}<0.05)$ and lower LDL cholesterol $(\mathrm{P}>0.05)$ of $\mathrm{CM}$ fed groups compared to the control indicated the positive effect of $\mathrm{CM}$ in lowering serum cholesterol content. CM may prevent the accumulation of LDL cholesterol probably by enriching the monounsaturated as well as the UFA which are considered the heart-friendly fatty acids to the consumer (Dernekbasi \& Karayucel, 2010).

\subsection{Fatty Acids Profile of Carcass Meat}

Chickens as a monogastric is able to incorporate the long chain fatty acids in the adipose tissue directly from the diet. Rahimi et al. (2011) concluded that fatty acids composition of the diet has an effect on fatty acids profile of broiler meat. In the present study, linoleic acid in carcass meat increased as dietary CM increased compared to the control. The results of this experiment are Compatible with the results obtained by Tuunainen et al. (2016); they found a significantly decrease in SFA and significantly increase in UFA of broiler breast meat that fed on rapeseed meal based diet compared to that fed SBM based diet. The increased values of linoleic and other UFA particularly palmitolic and oleic as mono UFA make the carcass meat a rich source of such favorable fatty acids for human consumption that attributed to its protection role of body human from heart diseases like heart attack and cardio vascular disorders (Rahimi et al., 2011).

\section{Conclusion}

Conclusively, there were no detrimental effects of using CM protein up to $60 \%$ replacement from SBM protein on productive performance and immune parameters. While, $90 \%$ had adversely effects on broiler chicks, likely because of increased dietary concentration of glucosinolates.

\section{Acknowledgements}

The authors are thankful for the research funds from Regional Centre for Food and Feed for supporting this project.

\section{References}

Abbas, T. E. (2013). The use of Moringa oleifera in poultry diets. Turkish Journal of Veterinary and Animal Sciences, 37(5), 492-496. https://doi.org/10.3906/vet-1211-40

Abdel-Ati, K. A., Latshaw, J. D., \& Donahoe, J. (1984). Distribution of selenium in chicken tissues as affected by bursectomy. Poultry Science, 63(3), 518-523. https://doi.org/10.3382/ps.0630518

Abou-Raya, A. K., \& Galal, A.-G. H. (1971). Evaluation of poultry feeds in digestion trials with reference to some factors involved. Egypt. J. Anim. Prod., 11, 207-221.

Aftab, U. (2009). Utilization of alternative protein meals with or without multiple-enzyme supplementation in broilers fed low-energy diets. Journal of Applied Poultry Research, 18(2), 292-296. https://doi.org/ 10.3382/japr.2008-00103

Ahmad, G., Mushtaq, T., Mirza, M. A., \& Ahmed, Z. (2007). Comparative Bioefficacy of Lysine from 1-Lysine Hydrochloride or l-Lysine Sulfate in Basal Diets Containing Graded Levels of Canola Meal for Female Broiler Chickens. Poultry Science, 86(3), 525-530. https://doi.org/10.1093/ps/86.3.525

Ahmed, H. A., Abou-Elkhair, R., Ketkat, S. A., \& Selim, S. (2015). Growth and Economic Performance of Broiler Chickens Fed on Graded Levels of Canola Meal with or without Multi-Enzyme Supplementation. Journal of Agricultural Science, 7(6), 137. https://doi.org/10.5539/jas.v7n6p137

Aljuobori, A., Zulkifli, I., Soleimani, A. F., Abdullah, N., Liang, J. B., \& Mujahid, A. (2016). Higher inclusion rate of canola meal under high ambient temperature for broiler chickens. Poultry Science, 95, 1326-1331. https://doi.org/10.3382/ps/pew023

An, B., Jung, J., Oh, S., Kang, C., Lee, K., \& Lee, S. (2016). Effects of Diets with Graded Levels of Canola Meal on the Growth Performance, Meat Qualities, Relative Organ Weights, and Blood Characteristics of Broiler Chickens. Revista Brasileira de Ciência Avícola, 18(2), 351-356. https://doi.org/10.1590/ 1806-9061-2015-0053 
AOAC (Association of Official Analytical Chemists). (2016). Association of Official Analytical Chemists of Official Methods of Analysis (20th ed.). Washington, D.C., USA.

Arbor Acres Plus Broiler Nutrition Specifications. (2014). Retrieved from http://www.aviagen.com

Ashnie, E., Mengistu, U., \& Aleme, A. (2015). Effects of Different Levels of Solvent Extracted Rapeseed (Brassica carinata) Meal Replacement to Soybean Meal on the Performance of Broiler Chicks. Food Science and Quality Management, 36.

Bovera, F., Nizza, S., Attia, Y. A., Di Meo, C., Piccolo, G., \& Nizza, A. (2014). Prediction of digestible energy and gross energy digestibility of feeds and diets in ostriches. British Poultry Science, 55, 518-523. https://doi.org/10.1080/00071668.2014.935702

Canola Council of Canada. (2015). Canola meal feed industry guide of Canola Council of Canada. Winnipeg, MB.

Collin, C. H., Lyne, P. M., \& Grange, J. M. (1995). Collin and Lyn's Microbiological Methods (p. 512). Butterworth Heinemann Ltd., Oxford. https://doi.org/ 10.1099/00222615-43-4-310b

Dernekbasi, S., \& Karayücel, I. (2010). Utilization of canola oil in fish feed. J. Fisheries Sci. Com., 4, 469-479. https://doi.org/10.3153/jfscom.2010051

Duncan, D. B. (1955). The multiple ranges and multiple F-Tests. Biometrics, 11, 1-42. https://doi.org/ $10.2307 / 3001478$

El-Medany, S. H. A., \& El-Reffaei, W. H. M. (2015). Evaluation Canola Meal on Growing Rabbits; Nutritionally and on Their Nutritional Meat Quality. J. Food and Nutrition Research, 3(4), 220-234.

Folch, J., Lees, M., \& Stanley, G. H. S. (1957). A simple method for the isolation and purification of total lipides from animal tissues. J. Biol. Chem., 226(1), 497-502.

Gadzirayi, C. T., Masamha, B., Mupangwa, J. F., \& Washaya, S. (2012). Performance of broiler chickens fed on mature moringa oleifera leaf meal as a protein supplement to soyabean meal. Int J Poultry Sci, 11, 5-10. https://doi.org/10.3923/ijps.2012.5.10

Gopinger, E., Xavier, E. G., Elias, M. C., Catalan, A. A. S., Castro, M. L. S., Nunes, A. P., \& Roll, V. F. B. (2014). The effect of different dietary levels of canola meal on growth performance, nutrient digestibility, and gut morphology of broiler chickens. Poultry Science, 93, 1130-1136. https://doi.org/10.3382/ps.2013-03426

Husak, R. L., Sebranek, J. G., \& Bregendahl, K. (2008). A survey of commercially available broilers marketed as organic, pasture-raised, and conventional broilers for cooked meat yields, meat composition, and relative value. Poultry Science, 87, 2367-2376. https://doi.org/10.3382/ps.2007-00294

Iheukwumere, F. C., Ndubuisi, E. C., Mazi, E. A., \& Onyekwere, M. U. (2008). Performance, nutrient utilization and organ characteristics of broilers fed cassava leaf meal (Manihot esculenta Crantz). Pakistan J Nutr, 7 , 13-16. https://doi.org/10.3923/pjn.2008.13.16

Kermanshahi, H., \& Abbasipour, A. R. (2006). Replacement value of soybean meal with rapeseed meal supplemented with or without a dietary NSP-degrading enzyme on performance, carcass traits and thyroid hormones of broiler chickens. Int J Poultry Sci, 5, 234-236. https://doi.org/10. 3923/ijps.2006.932.937

Khajali, F., \& Slominski, B. A. (2012). Factors that affect the nutritive value of canola meal for poultry. Poultry Science, 91, 2564-2575. https://doi.org/10.3382/ps.2012-02332

Kundu, A., Singh, D. P., Mohapatra, S. C., Dash, B. B., Moudgal, R. P., \& Bisht, G. S. (1999). Antibody response to sheep erythrocytes in Indian native vis-àvis imported breeds of chickens. British Poultry Science, 40(1), 40-44. https://doi.org/10.1080/00071669987818

Landero, J. L., Beltranena, E., Cervantes, M., Araiza, A. B., \& Zijlstra, R. T. (2012). The effect of feeding expeller-pressed canola meal on growth performance and diet nutrient digestibility in weaned pigs. Animal Feed Science and Technology, 171(2-4), 240-245. https://doi.org/10.1016/j.anifeedsci.2011.11.004

Lillehoj, H. S., \& Lillehoj, E. P. (2000). Avian coccidiosis. A review of acquired intestinal immunity and vaccination strategies. Avian Diseases, 44, 408-425. https://doi.org/10.2307/1592556

Mansoori, B., \& Acamovic, T. (2007). The effect of tannic acid on the excretion of endogenous methionine, histidine and lysine with broilers. Animal Feed Science and Technology, 134(3-4), 198-210. https://doi.org/ 10.1016/j.anifeedsci.2006.07.007 
Maroufyan, E., \& Kermanshahi, H. (2006). Effect of Different Levels of Rapeseed Meal Supplemented with Calcium Iodate on Performance, Some Carcass Traits, and Thyroid Hormones of Broiler Chickens. Int. J. Poultry Sci., 5, 1073-1078. https://doi.org/10.3923/ijps.2006.1073.1078

Mikulski, D., Jankowski, J., Zdunczyk, Z., Juskiewicz, J., \& Slominski, B. A. (2012). The effect of different dietary levels of rapeseed meal on growth performance, carcass traits, and meat quality in turkeys. Poultry Science, 91, 215-223. https://doi.org/10.3382/ps.2011-01587

Mnisi, C. M., \& Mlambo, V. (2018). Growth performance, haematology, serum biochemistry and meat quality characteristics of Japanese quails (Coturnix coturnix japonica) fed canola meal-based diets. Anim Nutr., 4, 37-43. https://doi.org/10.1016/j.aninu.2017.08.011

Moghaddam, H. N., Salari, S., Arshami, J., Golian, A., \& Maleki, M. (2012). Evaluation of the nutritional value of sunflower meal and its effect on performance, digestive enzyme activity, organ weight, and histological alterations of the intestinal villi of broiler chickens. J. Appl. Poult. Re., 21, 293-304. https://doi.org/ 10.3382/japr.2011-00396

Mushtaq, T., Sarwar, M., Ahmad, G., Mirza, M. A., Nawaz, H., Haroon Mushtaq, M. M., \& Noreen, U. (2007). Influence of canola meal-based diets supplemented with exogenous enzyme and digestible lysine on performance, digestibility, carcass, and immunity responses of broiler chickens. Poultry Science, 86, 2144-2151. https://doi.org/10.1093/ps/86.10.2144

Newkirk, R. (2009). Canola meal: Feed industry guide. Retrieved from http://www.canolacouncil.org/media/ 516716/canola_meal_feed_guide_english.pdf

Newkirk, R., \& Classen, H. (2002). The effects of toasting canola meal on body weight, feed conversion efficiency, and mortality in broiler chickens. Poultry Science, 81(6), 815-825. https://oi.org/10.1093/ $\mathrm{ps} / 81.6 .815$

Okuliarova, M., Groothuis, T. G. G., Skrobanek, P., \& Zeman, M. (2011). Experimental evidence for genetic heritability of maternal hormone transfer to offspring. Am. Nat., 177, 824-834. https://doi.org/10.1086/ 659996

Payvastegan, S., Farhoomand, P., \& Delfani, N. (2013). Growth performance, organ weights and, blood parameters of broilers fed diets containing graded levels of dietary canola meal and supplemental copper. Journal of Poultry Science, 50, 354-363. https://doi.org/10.2141/jpsa.0130006

Payvastegan, S., Farhoomand, P., Daneshyar, M., \& Ghaffari, M. (2017). Evaluation of Different Levels of Canola Meal on Performance, Organ Weights, Hepatic Deiodinase Gene Expression and Thyroid Morphology in Broiler Chickens. The Journal of Poultry Science, 54(4), 282-291. https://doi.org/ 10.2141/jpsa.0160147

Pustjens, A. M., Schols, H. A., Kabel, M. A., \& Gruppen, H. (2013). Characterisation of cell wall polysaccharides from rapeseed (Brassica napus) meal. Carb. Polymer., 98, 1650-1656. https://doi.org/ 10.1016/j.carbpol.2013.07.059

Rahimi, S., Azad, S. K., \& Torshizi, M. A. (2011). Omega-3 Enrichment of Broiler Meat by Using Two Oil Seeds. Journal of Agricultural Science and Technology, 13, 353-365.

Ravindran, V., Abdollahi, M. R., \& Bootwalla, S. M. (2014). Nutrient analysis, metabolizable energy and digestible amino acids of soybean meals of different regions for broilers. Poultry Science, 93, 2567-2577. https://doi.org/10.3382/ps.2014-04068

Ross, A. H., Van der Kamp, H. J., \& Marley, E. C. (1997). Comparison of immunoaffinity columns with Florisil/C18 columns for the determination of aflatoxins in animal feed and maize. Mycotoxin Research, 13, 2-10. https://doi.org/10.1007/bf02945056

SAS Institute Inc. (2004). SAS User's Guide: Statistics (Version 9.1). SAS Inst., Inc., Cary, NC., USA.

Slominski, B. A., \& Campbell, L. D. (1990). Non-starch polysaccharides of canola meal: Quantification, digestibility in poultry and potential benefit of dietary enzyme supplementation. Journal of the Science of Food and Agriculture, 53(2), 175-184. https://doi.org/10.1002/jsfa.2740530205

Swayne, D. E., Glisson, J. R., Jackwood, M. W., Pearson, J. E., \& Reed, W. M. (1998). A Laboratory Manual for the Isolation and Identification of Avian Pathogens (4th ed., pp. 74-80). Am. Assoc. Avian Pathol. Inc, Kennett Square, Pennsylvania, USA. 
Taraz, Z., Jalali, S., \& Rafeie, F. (2006). Effects of replacement of soybean meal with rapeseed meal on organs weight some blood biochemical parameters and performance of broiler chicks. International Journal of Poultry Science, 5, 1110-1115. https://doi.org/10.3923/ijps.2006.1110.1115

Tarek, M. S., Hamad, A. A.-B., \& Ahmed, M. S. F. (2015). The Effect of Dietary Flaxseed Meal on Liver and Egg Yolk Fatty Acid Profiles, Immune Response and Antioxidant Status of Laying Hens, Italian Journal of Animal Science, 14(3), 3939. https://doi.org/10.4081/ ijas.2015.3939

Thacker, P. A., \& Petri, D. (2011). Nutritional Evaluation of Canola Protein Concentrate for Broiler Chickens. Asian-Australasian Journal of Animal Sciences, 24(11), 1607-1614. https://doi.org/10.5713/ajas.2011.11161

Toghyani, M., Wu, S. B., Pérez-Maldonado, R. A., Iji, P. A., \& Swick, R. A. (2017). Performance, nutrient utilization, and energy partitioning in broiler chickens offered high canola meal diets supplemented with multicomponent carbohydrase and mono-component protease. Poultry Science, 96(11), 3960-3972. https://doi.org/10.3382/ps/pex212

Tuunainen, P., Koivunen, E., Valaja, J., Valkonen, E., Hiidenhovi, J., \& Tupasela, T. (2016). Effects of dietary rapeseed meal and peas on the performance and meat quality of broilers. Agricultural and Food Science, 25(1), 22-33. https://doi.org/10.23986/afsci.52299

USDA. (2014). Oil seeds (Table 17: World: Rapeseed and Products Supply and Distribution). Foreign Agricultural Service, USDA.

Wood, J. D., Enser, M., Fisher, A. V., Nute, G. R., Sheard, P. R., \& Richardson, R. I. (2008). Fat deposition, fatty acid composition and meat quality: A review. Meat Science, 78, 343-353. https://doi.org/10.1016/ j.meatsci.2007.07.019

Woyengo, T. A., Kiarie, E., \& Nyachoti, C. M. (2011). Growth performance, organ weights, and blood parameters of broilers fed diets containing expeller-extracted canola meal. Poultry Science, 90, 2520-2527. https://doi.org/10.3382/ps.2011-01436

Yuan, C., Song, H., Zhang, X., Jiang, Y., Zhang, A., Azzam, M. M., \& Zou, X. (2014). Effect of expanded cottonseed meal on laying performance, egg quality, concentrations of free gossypol in tissue, serum and egg of laying hens. Animal Science Journal, 85(5), 549-554. https://doi.org/10.1111/asj.12169

Zhang, F., \& Adeola, O. (2017). Energy values of canola meal, cottonseed meal, bakery meal, and peanut flour meal for broiler chickens determined using the regression method. Poultry Science, 96(2), 397-404. https://doi.org/10.3382/ps/pew239

\section{Copyrights}

Copyright for this article is retained by the author(s), with first publication rights granted to the journal.

This is an open-access article distributed under the terms and conditions of the Creative Commons Attribution license (http://creativecommons.org/licenses/by/4.0/). 